KYUNGPOOK Math. J. 56(2016), 1237-1246

http://dx.doi.org/10.5666/KMJ.2016.56.4.1237

pISSN 1225-6951 eISSN 0454-8124

(c) Kyungpook Mathematical Journal

\title{
On Some Properties of Riemannian Manifolds with a Gener- alized Connection
}

\author{
Azam Etemad DehKordy \\ Department of Mathematical Sciences, Isfahan University of Technology, 84156- \\ 83111, Isfahan, Iran \\ e-mail: ae110mat@cc.iut.ac.ir
}

ABSTRACT. In this paper we study some properties of submanifolds of a Riemannian manifold equipped with a generalized connection $\widehat{\nabla}$. We also consider almost Hermitian manifolds that admits a special case of this generalized connection and derive some results about the behavior of this manifolds.

\section{Introduction}

The Levi-Civita connection $\bar{\nabla}$ is an ideal connection on a Riemannian manifold $(M, g)$ that gives many facilities for calculus on manifolds. However, there are many other connections $\tilde{\nabla}$ on $(M, g)$ with less abilities.

The notion of semi-symmetric linear connection on a diferential manifold was introduced in [6]. Hayden also defined and studied subspaces of space with torsion or semi-symmetric metric connection in [8]. Later, a number of authors published many papers on this topic until now (for example see [7], [9], [10]). Some of them introduced another connections such as quarter-symmetric recurrent connection, semi-symmetric recurrent metric connection, Weyl connection and vice versa.

Tripathi in [11] introduced a new connection which unifies all the previously connections and some other connections not introduced so far. He also derived curvature tensor of this connection.

This paper has two main purposes. The first is to extend the some results of [5] about submanifolds of a Riemannian manifold with a semi-symmetric nonmetric connections to the these submanifolds with the generalized connection which introduced in [11].

Received February 14, 2016; accepted July 13, 2016

2010 Mathematics Subject Classification: 53C60, 53C44.

Key words and phrases: Generalized connection; submanifold; almost Hermitian; Kähler manifold.

This work was supported by Isfahan university of Technology. 
Secondly, almost hermitian manifolds and because of their nice topological properties Kähler mnifolds have been studied extensively (see for example [2],[3]). Thus it is worthwhile to study some properties of almost hermitian manifolds which endowed by this generalized connection.

The torsion tensor $\widetilde{T}$ of a given connection $\widetilde{\nabla}$ on $M$, is defined by

$$
\widetilde{T}(X, Y)=\widetilde{\nabla}_{X} Y-\widetilde{\nabla}_{Y} X-[X, Y]
$$

The connection $\widetilde{\nabla}$ is called symmetric or torsion-free if $\widetilde{T} \equiv 0$, otherwise it is called non-symmetric. The connection $\widetilde{\nabla}$ is called compatible with metric or briefly metric if $\tilde{\nabla} g=0$, otherwise it is called non-metric. As we know, the Levi-Civita connection $\bar{\nabla}$ is a metric and symmetric connection. However, there are connections which are either non-symmetric or non-metric (see for example [1], [4], [9]).

Let $k_{1}, k_{2}$ be non-zero real functions on $M, \eta, \eta_{1}, \eta_{2}$ are 1 -forms on $M$ and $\psi$ is a $(1,1)$ tensor field on $M$. Let

$$
\begin{gathered}
\eta(X)=g(E, X), \eta_{1}(X)=g\left(E_{1}, X\right), \eta_{2}(X)=g\left(E_{2}, X\right), \\
g(\psi X, Y)=\Psi(X, Y)=\Psi_{1}(X, Y)+\Psi_{2}(X, Y)
\end{gathered}
$$

where $\Psi_{1}$ and $\Psi_{2}$ are symmetric and skew-symmetric parts of the $(0,2)$ tensor $\Psi$ such that

$$
\Psi_{1}(X, Y)=g\left(\psi_{1} X, Y\right), \quad \Psi_{2}(X, Y)=g\left(\psi_{2} X, Y\right) .
$$

Considering $\left\{k_{1}, k_{2}, \eta, \eta_{1}, \eta_{2}, \psi\right\}$, Tripathi in Theorem 2.1 of [11], proved that there is a unique connection $\widetilde{\nabla}$ on $M$ given by

$$
\begin{aligned}
\tilde{\nabla}_{X} Y= & \bar{\nabla}_{X} Y+\eta(Y) \psi_{1} X-\eta(X) \psi_{2} Y-g\left(\psi_{1} X, Y\right) E-k_{1}\left\{\eta_{1}(X) Y+\right. \\
& \left.\eta_{1}(Y) X-g(X, Y) E_{1}\right\}-k_{2} g(X, Y) E_{2}
\end{aligned}
$$

with

$$
\widetilde{T}[X, Y]=\eta(Y) \psi X-\eta(X) \psi Y
$$

and

$$
\left(\widetilde{\nabla}_{X} g\right)(Y, Z)=2 k_{1} \eta_{1}(X) g(Y, Z)+k_{2}\left\{\eta_{2}(Y) g(X, Z)+\eta_{2}(Z) g(X, Y)\right\} .
$$

This connection is a generalized connection because in special cases, we obtain other well-known connections such as quarter-symmetric metric connections, semisymmetric metric S-connection, Weyl connection and etc. [11].

\section{Submanifolds of a Riemannian Manifold with a Generalized Connec- tion}

Let $M$ be an $n$-dimensional submanifold of an $(n+k)$-dimensional Riemannian manifold $(\widetilde{M}, g)$. With the Levi-Civita connection $\bar{\nabla}$ on $\widetilde{M}$, we have the Gauss 
formula as follows,

$$
\bar{\nabla}_{X} Y=\bar{\nabla}_{X} Y+h(X, Y)
$$

where $X, Y \in T M$ and $\bar{\nabla}$ induced Rimannian connection on $M$. Now consider $\widetilde{M}$ with the generalized connection $\widetilde{\nabla}$, that is introduced in (1.1). Let $\nabla$ be the induced connection from the $\widetilde{\nabla}$ on $M$ and $\widetilde{h}$ is the second fundamental form of $M$ in $\widetilde{M}$. By using notations above, we define a formula similar to Gauss's, as follows: $(2.2)$

$$
\widetilde{\nabla}_{X} Y=\nabla_{X} Y+\widetilde{h}(X, Y)
$$

where $X, Y$ are $T M$. The mean curvature vector of $M$ with respect to Levi-Civita connection $\bar{\nabla}$ and $\widetilde{\nabla}$ respectively defined as $H=\frac{1}{n}$ trace $h$ and $\widetilde{H}=\frac{1}{n}$ trace $\widetilde{h}$.

More precisely, let $\left\{e_{1}, e_{2}, \ldots, e_{n}\right\}$ be an orthonormal basis of the tangent space of $M$, so

$$
H=\frac{1}{n} \sum_{i=1}^{n} h\left(e_{i}, e_{i}\right), \quad \widetilde{H}=\frac{1}{n} \sum_{i=1}^{n} \widetilde{h}\left(e_{i}, e_{i}\right)
$$

If $\widetilde{h}=0$ then $M$ is called a totally geodesic submanifold of $\widetilde{M}$ with respect to $\widetilde{\nabla}$. If $\widetilde{H}=0$ then $M$ is called a minimal submanifold of $\widetilde{M}$ with respect to $\widetilde{\nabla}$.

Furthermore if $\widetilde{h}(X, Y)=g(X, Y) H$ for any tangent vectors $X, Y$, then $M$ is called a totally umbilical submanifold of $\widetilde{M}$ with respect to $\widetilde{\nabla}$.

In this paper we use notations $X, Y, Z$ for tangent vector fields to $M$ and notations $\zeta, \nu$ for normal vector fields on $M$.

The vector fields $E_{1}, E_{2}, E_{3}$ and $E$ on $M$ decomposed uniquely into tangent components $E_{1}^{\top}, E_{2}^{\top}, E_{3}^{\top}$ and $E^{\top}$ and normal components $E_{1}^{\perp}, E_{2}^{\perp}, E_{3}^{\perp}$ and $E^{\perp}$, respectively.

Using definition of generalized connections $\tilde{\nabla},(2.1)$ and (2.2), we get

$$
\begin{aligned}
\nabla_{X} Y= & \bar{\nabla}_{X} Y+\eta(Y)\left(\psi_{1} X\right)^{\top}-\eta(X)\left(\psi_{2} Y\right)^{\top}-g\left(\psi_{1} X, Y\right) E^{\top} \\
& -k_{1}\left\{\eta_{1}(X) Y+\eta_{1}(Y) X-g(X, Y) E_{1}^{\top}\right\}-k_{2} g(X, Y) E_{2}^{\top} \\
\widetilde{h}(X, Y)= & h(X, Y)+\eta(Y)\left(\psi_{1} X\right)^{\perp}-\eta(X)\left(\psi_{2} Y\right)^{\perp}-g\left(\psi_{1} X, Y\right) E^{\perp} \\
& +k_{1} g(X, Y) E_{1}^{\perp}-k_{2} g(X, Y) E_{2}^{\perp}
\end{aligned}
$$

Using (2.1) we have the following Theorems.

Theorem 2.1. Let $\widetilde{M}$ be a Riemannian manifold admits the generalized connection $\widetilde{\nabla}$ in (1.1). Suppose that $M$ is a submanifold of $\widetilde{M}$ with connection $\nabla$ induced from $\widetilde{\nabla}$. Then $\nabla$ is the generalized connection(1.1) on $M$.

As a direct consequence of 2.4 , we obtain the following theorem.

Theorem 2.2. Let $\widetilde{M}$ be a Riemannian manifold equipped with the generalized connection $\widetilde{\nabla}$ in 1.1. Suppose that $M$ is a submanifold of $\widetilde{M}$ with connection $\nabla$ induced from $\tilde{\nabla}$.

Furthermore, let $E_{1}, E_{2}, E, \psi_{1} X, \psi_{2} X, \psi X$ be tangent to $M$ for all $X \in T M$. Then $M$ is a totally geodesic (respectively, totally umbilical) submanifold with respect to the Levi-Cevita connection if and only if $M$ is a totally geodesic (respectively, totally umbilical) submanifold with respect to $\tilde{\nabla}$. Furthermore, $M$ is a minimal 
submanifold with respect to the Levi-Cevita connection if and only if $M$ is a minimal submanifold with respect to $\widetilde{\nabla}$.

Remark 2.3. In some special cases, for example one case of semi-symmetric recurrent metric connections or one case of semi-symmetric non-metric connection without any restriction, $M$ is a totally geodesic (respectively, totally umbilical) submanifold with respect to the Levi-Cevita connection if and only if $M_{\sim}$ is a totally geodesic (respectively, totally umbilical) submanifold with respect to $\widetilde{\nabla}$. Furthermore, $M$ is a minimal submanifold with respect to the Levi-Cevita connection if and only if $M$ is a minimal submanifold with respect to $\widetilde{\nabla}$.

Let $\zeta$ be a normal vector field on $M$. We have

$\tilde{\nabla}_{X} \zeta=\bar{\nabla}_{X} \zeta+\eta(\zeta) \psi_{1} X-\eta(X) \psi_{2} \zeta-g\left(\psi_{1} X, \zeta\right) E-k_{1}\left\{\eta_{1}(X) \zeta-\eta_{1}(\zeta) X\right\}$.

We know that $\bar{\nabla}_{X} \zeta=-\bar{A}_{\zeta} X+\bar{\nabla}_{X}^{\perp} \zeta$, where $\bar{A}_{\zeta}$ is the shape operator of $M$ in the direction of $\zeta$. If we assume $\psi_{1} X$ is tangent to $M$, for all $X \in T M$ and $\psi_{2} \zeta$ is normal to $M$ for all normal vector field $\zeta$ on $M$ then we can consider a $(1,1)$ tensor $\widetilde{A}_{\zeta}$ on $M$ in the direction of $\zeta$ and with respect to the generalized connection $\tilde{\nabla}$ that define as follows.

$$
\widetilde{A}_{\zeta}=\bar{A}_{\zeta}-\eta(\zeta) \psi_{1}-k_{1} \eta_{1}(\zeta) I
$$

Therefore we can define Weingarten formulas with respect to the generalized connection $\widetilde{\nabla}$ as follows.

$$
\widetilde{\nabla}_{X} \zeta=-\widetilde{A}_{\zeta} X+\bar{\nabla}_{X}^{\perp} \zeta-\eta(X) \psi_{2} \zeta-k_{1} \eta_{1}(X) \zeta
$$

It can be easily seen for all generalized connections, $g\left(\widetilde{A}_{\zeta} X, Y\right)=g\left(X, \widetilde{A}_{\zeta} Y\right)$ In the general case, we have

$$
\left.g\left(\left[\widetilde{A}_{\zeta}, \widetilde{A}_{\nu}\right] X, Y\right)=g\left(\left[\bar{A}_{\zeta}, \bar{A}_{\nu}\right] X, Y\right)+\eta(\nu) g\left(\alpha_{\zeta} X, Y\right)+\eta(\zeta) g\left(\alpha_{\nu} X, Y\right)\right) .
$$

where $\alpha_{\zeta}=\bar{A}_{\zeta} \psi_{1}-\psi_{1} \bar{A}_{\zeta}$ for unit normal vector field $\zeta$ on $M$.

From now on, we consider for simplicity the cases that $\psi_{1} X$ is tangent to $M$, for all $X \in T M$ and $\psi_{2} \zeta$ is normal to $M$ for all normal vector field $\zeta$ on $M$.

Now we plan to study tensor curvature of a submanifold $M^{n}$ of a Riemannian manifold $\widetilde{M}^{n+k}$ that equipped with the generalized connection $\widetilde{\nabla}$.

Let $\widetilde{R}$ and $R$ denote, respectively, the curvature tensor of $\widetilde{M}$ with respect to the generalized connection $\widetilde{\nabla}$ and the curvature tensor of $M$ with respect to induced connection $\nabla$ of $\widetilde{\nabla}$, so we have

$$
\begin{aligned}
\widetilde{R}(X, Y) Z & =\tilde{\nabla}_{X} \tilde{\nabla}_{Y} Z-\tilde{\nabla}_{Y} \tilde{\nabla}_{X} Z-\widetilde{\nabla}_{[X, Y]} Z \\
R(X, Y) Z & =\nabla_{X} \nabla_{Y} Z-\nabla_{Y} \nabla_{X} Z-\nabla_{[X, Y]} Z
\end{aligned}
$$

where $X, Y, Z \in T M$. Substituting (2.2) in $\widetilde{R}(X, Y) Z$ and use of (2.7), after simplification we have,

$$
\begin{aligned}
& \widetilde{R}(X, Y) Z=R(X, Y) Z+\widetilde{h}\left(X, \nabla_{Y} Z\right)-\widetilde{h}\left(Y, \nabla_{X} Z\right)-\widetilde{h}([X, Y], Z)- \\
& A_{\widetilde{h}(Y, Z)} X+A_{\widetilde{h}(X, Z)} Y+\nabla_{X}^{\perp} \widetilde{h}(Y, Z)-\nabla_{Y}^{\perp} \widetilde{h}(X, Z)- \\
& \eta(X) \psi_{2} \widetilde{h}(Y, Z)+\eta(Y) \psi_{2} \widetilde{h}(X, Z)-k_{1} \eta_{1}(X) \widetilde{h}(Y, Z) \\
& +k_{1} \eta_{1}(Y) \widetilde{h}(X, Z)
\end{aligned}
$$


Since we assume that $\psi_{1} X$ is tangent to $M$, for all $X \in T M,(2.4)$ reduced to $(2.9) \widetilde{h}(X, Y)=h(X, Y)-\eta(X) \psi_{2} Y-g\left(\psi_{1} X, Y\right) E^{\perp}+k_{1} g(X, Y) E_{1}^{\perp}-k_{2} g(X, Y) E_{2}^{\perp}$ Since $g\left(\psi_{2} Z, \zeta\right)=g\left(Z, \psi_{2} \zeta\right)$ for every normal vector field $\zeta$ on $M$, hence, from (2.8) and (2.9) we reach to the following equation.

$$
\begin{aligned}
\widetilde{R}(X, Y, Z, W) & =R(X, Y, Z, W)-g(h(Y, Z), h(X, W))+g(h(X, Z), h(Y, W)) \\
& -k_{1}\left\{g(Y, Z) \eta_{1}(h(X, W))-g(X, Z) \eta_{1}(h(Y, W))\right\} \\
& +k_{2}\left\{g(Y, Z) \eta_{2}(h(X, W))-g(X, Z) \eta_{2}(h(Y, W))\right\} \\
& +g\left(\psi_{1} Y, Z\right) \eta_{1}(h(X, W))-g\left(\psi_{1} X, Z\right) \eta_{1}(h(Y, W))
\end{aligned}
$$

Now we can compute the relation between sectional curvatures of $\widetilde{M}$ and $M$ when we consider the generalized connection $\widetilde{\nabla}$ on $\widetilde{M}$. Let $X$ and $Y$ be orthonormal unit tangent vector fields on $M$, so by (2.10), we have

$$
\begin{aligned}
\widetilde{R}(X, Y, Y, X) & =R(X, Y, Y, X)-g(h(Y, Y), h(X, X))+g(h(X, Y), h(Y, X)) \\
& +\eta(Y) g\left(\psi_{2} Y, h(X, X)\right)-\eta(X) g\left(\psi_{2} Y, h(Y, X)\right)- \\
& k_{1}\left\{g(Y, Y) \eta_{1}(h(X, X))-g(X, Y) \eta_{1}(h(Y, X))\right\}+ \\
& k_{2}\left\{g(Y, Y) \eta_{2}(h(X, X))-g(X, Y) \eta_{2}(h(Y, X))\right\}+ \\
& g\left(\psi_{1} Y, Y\right) \eta_{1}(h(X, X))-g\left(\psi_{1} X, Y\right) \eta_{1}(h(Y, X))
\end{aligned}
$$

Furthermore by assumptions $g\left(\psi_{2} X, \zeta\right)=-g\left(X, \psi_{2} \zeta\right)=0$, so we get

$$
\begin{aligned}
\widetilde{R}(X, Y, Y, X) & =R(X, Y, Y, X)-g(h(Y, Y), h(X, X))+g(h(X, Y), h(Y, X)) \\
& -k_{1} \eta_{1}(h(X, X))+k_{2} \eta_{2}(h(X, X))+g\left(\psi_{1} Y, Y\right) \eta_{1}(h(X, X)) \\
& -g\left(\psi_{1} X, Y\right) \eta_{1}(h(Y, X))
\end{aligned}
$$

Therefore we obtain the following formula for relation between sectional curvature $K$ of $M$ and sectional curvature $\widetilde{K}$ of $\widetilde{M}$ with respect to the generalized connection.

$$
\begin{aligned}
\widetilde{K}(X, Y) & =K(X, Y)-g(h(Y, Y), h(X, X))+g(h(X, Y), h(Y, X)) \\
& -k_{1} \eta_{1}(h(X, X))+k_{2} \eta_{2}(h(X, X))+g\left(\psi_{1} Y, Y\right) \eta_{1}(h(X, X)) \\
& -g\left(\psi_{1} X, Y\right) \eta_{1}(h(Y, X))
\end{aligned}
$$

In the special case when $\psi_{1}=0$, we have

$$
\begin{gathered}
\widetilde{K}(X, Y)=K(X, Y)-g(h(Y, Y), h(X, X))+g(h(X, Y), h(X, Y))- \\
k_{1} \eta_{1}(h(X, X))+k_{2} \eta_{2}(h(X, X))
\end{gathered}
$$

Moreover, if $E_{1}$ and $E_{2}$ are tangent vector fields to $M$ then,

$$
\widetilde{K}(X, Y)=K(X, Y)-g(h(Y, Y), h(X, X))+g(h(X, Y), h(X, Y))
$$

Finally, we have the following theorem.

Theorem 2.4. Let $M$ be a submanifold of a Riemannian manifold $\widetilde{M}$ admitting the generalized connection $\widetilde{\nabla}$. Moreover, let for $I \subseteq R, \lambda: I \rightarrow M \subset \widetilde{M}$ be a 
geodesic, $U$ be the unit tangent vector field of $\lambda$ in $M$ and $X$ tangent vector fields to $M$. If $E_{1}$ is tangent to $M$ then $\widetilde{K}(U, X) \geq K(U, X)$. Furthermore, if $X$ is a unit tangent vector field which is parallel along $\lambda$ in $M$ and orthogonal to $U$ then the equality holds if and only if $X$ is parallel along $\lambda$ in $\widetilde{M}$.

\section{Some Properties of the Generalized Connection on Almost Hermitian Manifold}

Let $\widetilde{M}$ be an even dimensional $C^{r}$ Riemannian manifold. An almost complex manifold $\widetilde{M}$ is defined by a structure tensor $\mathrm{J}$ of type $(1,1)$, satisfying $J^{2} X=-X$ for any vector field $\mathrm{X}$ on $\mathrm{M}$.

Furthermore, if $\widetilde{M}$ endowed with a non singular Hermitian metric $g$, i.e.

$$
g(J X, J Y)=g(X, Y)
$$

for every vector fields $X$ and $Y$ on $M$, then it is called an almost Hermitian manifold with almost Hermitian structure $\{J, g\}$.

If the exterior derivative $d J$ of $J$ vanishes, then $\mathrm{M}$ is called an almost Kähler manifold. Further, if $\bar{\nabla} J=0$ for the Levi-Civita connection $\bar{\nabla}$ that defined by $g$, then $\mathrm{M}$ is called a Kähler manifold.

In this section, we consider an special case corresponds to the case $\psi=\psi_{1}=$ $\psi_{2}=I d$ of generalized connection and denote it by $\breve{\nabla}$.

$$
\begin{aligned}
\breve{\nabla}_{X} Y= & \bar{\nabla}_{X} Y+\eta(Y) X-\eta(X) Y-g(X, Y) E-k_{1}\left\{\eta_{1}(X) Y+\right. \\
& \left.\eta_{1}(Y) X-g(X, Y) E_{1}\right\}-k_{2} g(X, Y) E_{2}
\end{aligned}
$$

We will call this connection as $S$-generalized connection.

Theorem 3.1. Let $\widetilde{M}$ be an almost Hermitian manifold which admits $S$-generalized connection $\breve{\nabla}$. Then $\widetilde{M}$ is a Hermitian manifold if and only if

$$
\left(\breve{\nabla}_{J X} J\right)(J Y)=\left(\breve{\nabla}_{X} J\right)(Y)
$$

Proof. By covariant derivative of $J$ with respect to connection $\widetilde{\nabla}$, we have

$$
\left(\breve{\nabla}_{X} J\right)(Y)=\breve{\nabla}_{X} J Y-J\left(\breve{\nabla}_{X} Y\right) \text {. }
$$

Now from definition of generalized connection, (3.2) and linearity of $J$, we get,

$$
\begin{aligned}
\breve{\nabla}_{X} J Y= & \bar{\nabla}_{X} J Y+\eta(J Y) X-\eta(X) J Y-g(X, J Y) E+k_{1}\left\{\eta_{1}(X) J Y+\right. \\
& \left.\eta_{1}(J Y) X-g(X, J Y) E_{1}\right\}-k_{2} g(X, J Y) E_{2}
\end{aligned}
$$

and

$$
\begin{aligned}
J\left(\breve{\nabla}_{X} Y\right)= & J\left(\bar{\nabla}_{X} Y\right)+\eta(Y) J X-\eta(X) J Y-g(X, Y) J E+k_{1}\left\{\eta_{1}(X) J Y+\right. \\
& \left.\eta_{1}(Y) J X-g(X, Y) J E_{1}\right\}-k_{2} g(X, Y) J E_{2}
\end{aligned}
$$


So after simplification we obtain

$$
\begin{aligned}
\left(\breve{\nabla}_{X} J\right)(Y)= & \left(\bar{\nabla}_{X} J\right)(Y)+\eta(J Y) X-\eta(Y) J X-g(X, J Y) E+g(X, Y) J E \\
& +k_{1}\left\{\eta_{1}(J Y) X-\eta_{1}(Y) J X-g(X, J Y) E_{1}+g(X, Y) J E_{1}\right\} \\
& -k_{2}\left\{g(X, J Y) E_{2}-g(X, Y) J E_{2}\right\}
\end{aligned}
$$

In similar way, we have

$\left(\breve{\nabla}_{J X} J\right)(J Y)=\left(\bar{\nabla}_{J X} J\right)(J Y)+\eta\left(J^{2} Y\right) X-\eta(Y) J^{2} X-g\left(J X, J^{2} Y\right) E+$

$$
\begin{aligned}
& g(J X, J Y) J E+k_{1}\left\{\eta_{1}\left(J^{2} Y\right) J X-\eta_{1}(J Y) J^{2} X-g\left(J X, J^{2} Y\right)\right. \\
& \left.E_{1}+g(J X, J Y) J E_{1}\right\}-k_{2}\left\{g\left(J X, J^{2} Y\right) E_{2}-g(J X, J Y) J E_{2}\right\}
\end{aligned}
$$

By using relations $J^{2} X=-X$ and $g(J X, Y)=-g(X, J Y)$ for every vector fields $X, Y$ on $\widetilde{M}$, subtracting (3.3) from (3.4), gives us

$$
\left(\breve{\nabla}_{J X} J\right)(J Y)-\left(\breve{\nabla}_{X} J\right)(Y)=\left(\bar{\nabla}_{J X} J\right)(J Y)-\left(\bar{\nabla}_{X} J\right)(Y)
$$

But an almost Hermitian manifold is a Hermitian manifold, if and only if the righthand side of equation (3.5) is zero. So we have the result.

If we define $\widetilde{J}(X, Y)=g(J X, Y)$ for vector fields $X$ and $Y$ on $\widetilde{M}$, then

$$
\widetilde{J}(J X, J Y)=g\left(J^{2} X, J Y\right)=-g(X, J Y)=g(J X, Y)=\widetilde{J}(X, Y) .
$$

Now, if we set

$$
\begin{gathered}
\widetilde{\tau}(X, Y, Z)=\left(\breve{\nabla}_{X} \widetilde{J}\right)(Y, Z)+\left(\breve{\nabla}_{Y} \widetilde{J}\right)(Z, X)+\left(\breve{\nabla}_{Z} \widetilde{J}\right)(X, Y), \\
\bar{\tau}(X, Y, Z)=\left(\bar{\nabla}_{X} \widetilde{J}\right)(Y, Z)+\left(\bar{\nabla}_{Y} \widetilde{J}\right)(Z, X)+\left(\bar{\nabla}_{Z} \widetilde{J}\right)(X, Y), \\
\mu(X, Y, Z)=\left(\eta(X)+k_{1} \eta_{1}(X)\right) \widetilde{J}(Y, Z),
\end{gathered}
$$

then we have the following theorem.

Theorem 3.2. Let $\widetilde{J}$ is closed in an almost Hermitian manifold with respect to both S-generalized connection $\breve{\nabla}$ and Levi-Civita connection $\bar{\nabla}$. So we have

$$
\widetilde{\tau}(X, Y, Z)=4\{\mu(X, Y, Z)+\mu(Y, Z, X)+\mu(Z, X, Y)\} .
$$

Proof. By definition, we have

$$
\left(\breve{\nabla}_{X} \widetilde{J}\right)(Y, Z)=\left(\bar{\nabla}_{X} \widetilde{J}\right)(Y, Z)-\widetilde{J}\left(\breve{\nabla}_{X} Y-\bar{\nabla}_{X} Y, Z\right)-\widetilde{J}\left(Y, \breve{\nabla}_{X} Z-\bar{\nabla}_{X} Z\right) .
$$

But, (1.1) implies that

$$
\begin{aligned}
\left(\breve{\nabla}_{X} \widetilde{J}\right)(Y, Z)= & (\bar{\nabla} X \widetilde{J})(Y, Z)-\eta(Y) \widetilde{J}(X, Z)+\eta(X) \widetilde{J}(Y, Z)+ \\
& g(X, Y) \widetilde{J}(E, Z)+k_{1}\left\{\eta_{1}(X) \widetilde{J}(Y, Z)+\eta_{1}(Y) \widetilde{J}(X, Z)-\right. \\
& \left.g(X, Y) \widetilde{J}\left(E_{1}, Z\right)\right\}+k_{2} g(X, Y)\left(E_{2}, Z\right)-\eta(Z) \widetilde{J}(Y, X) \\
& +\eta(X) \widetilde{J}(Y, Z)+g(X, Z) \widetilde{J}(Y, E)+k_{1}\left\{\eta_{1}(X) \widetilde{J}(Y, Z)+\right. \\
& \left.\eta_{1}(Z) \widetilde{J}(Y, X)-g(X, Z) \widetilde{J}\left(Y, E_{1} Z\right)\right\}+k_{2} g(X, Z)\left(Y, E_{2}\right) .
\end{aligned}
$$


We use the circular permutation $X, Y, Z$ on (3.6) to obtain,

$$
\widetilde{\tau}(X, Y, Z)=\bar{\tau}(X, Y, Z)+4\{\mu(X, Y, Z)+\mu(Y, Z, X)+\mu(Z, X, Y)\} .
$$

Now, since $\widetilde{J}$ is closed with respect to $\bar{\nabla}$, i.e. $\bar{\tau}(X, Y, Z)=0$, so the proof is complete.

Theorem 3.3. The Nijenhuis tensors of S-generalized connection $\breve{\nabla}$ and LeviCivita connection $\bar{\nabla}$ on an almost Hermitian manifold are coincide.

Proof. By applying the definition of Nijenhuis tensor with respect to Levi-Civita connection on the almost Hermitian manifold, we have

But by using (1.1), we have

$$
\begin{aligned}
J\left(\left(\bar{\nabla}_{Y} J\right) X\right)= & J\left(\left(\breve{\nabla}_{Y} J\right)(X)\right)-\eta(J X) J Y-\eta(X) Y+g(Y, J X) J E+ \\
& g(X, Y) E-k_{1}\left\{\eta_{1}(J X) J Y-\eta_{1}(X) Y-g(J X, Y) J E_{1}\right. \\
& \left.-g(X, Y) E_{1}\right\}+k_{2}\left\{g(J X, Y) J E_{2}+g(X, Y) E_{2}\right\} \\
J\left(\left(\bar{\nabla}_{X} J\right) Y\right)= & J\left(\left(\breve{\nabla}_{X} J\right)(Y)\right)-\eta(J Y) J X-\eta(Y) X+g(X, J Y) J E+ \\
& g(X, Y) E-k_{1}\left\{\eta_{1}(J Y) J X-\eta_{1}(Y) X-g(X, J Y) J E_{1}\right. \\
& \left.-g(X, Y) E_{1}\right\}+k_{2}\left\{g(X, J Y) J E_{2}+g(X, Y) E_{2}\right\} \\
\left(\bar{\nabla}_{J X} J\right) Y= & \left(\breve{\nabla}_{J X} J\right)(Y)-\eta(J Y) J X-\eta(Y) X+g(J X, J Y) J E+ \\
& g(J X, Y) E-k_{1}\left\{\eta_{1}(J Y) J X-\eta_{1}(Y) X-g(J X, J Y) E_{1}\right. \\
& \left.+g(X, Y) J E_{1}\right\}+k_{2}\left\{g(J X, J Y) E_{2}-g(J X, Y) J E_{2}\right\} \\
\left(\bar{\nabla}_{J Y} J\right) X= & \left(\breve{\nabla}_{J Y} J\right)(X)-\eta(J X) J Y-\eta(X) Y+g(J X, J Y) J E+ \\
& g(J Y, X) E-k_{1}\left\{\eta_{1}(J X) J Y-\eta_{1}(X) Y-g(J Y, J X) E_{1}\right. \\
& \left.+g(X, Y) J E_{1}\right\}+k_{2}\left\{g(J Y, J X) E_{2}-g(J Y, X) J E_{2}\right\}
\end{aligned}
$$

Now by substituting the above terms into (3.7), we get

$$
\bar{N}(X, Y)=J\left(\left(\breve{\nabla}_{Y} J\right) X\right)-J\left(\left(\breve{\nabla}_{X} J\right) Y\right)+\left(\breve{\nabla}_{J X} J\right) Y-\left(\breve{\nabla}_{J Y} J\right) X=\breve{N}(X, Y),
$$

where $\breve{N}(X, Y)$ is the Nijenhuis tensor with respect to S-generalized connection.

Therefore we have the following corollary.

Corollary 3.4. If $\widetilde{M}$ is a Kähler Manifold that admits the S-generalized connection, then $\widetilde{M}$ is also a Kähler manifold with respect to the S-generalized connection.

For abbreviation, we set

$$
\begin{aligned}
\varrho(X, Y)= & \eta(J Y) X-\eta(Y) J X-g(X, J Y) E+g(X, Y) J E \\
& +k_{1}\left\{\eta_{1}(J Y) X-\eta_{1}(Y) J X-g(X, J Y) E_{1}+g(X, Y) J E_{1}\right\} \\
& -k_{2}\left\{g(X, J Y) E_{2}-g(X, Y) J E_{2}\right\} .
\end{aligned}
$$


So from definition of Kähler manifold and (3.5), we have the following obvious result.

Corollary 3.5. Let $\widetilde{M}$ be a Kähler manifold that admits the S-generalized connection. Then $\left(\breve{\nabla}_{J X} J\right)(J Y)=\left(\breve{\nabla}_{X} J\right)(Y)$. Moreover, $\left(\breve{\nabla}_{X} J\right) Y=0$ if and only if $\varrho(X, Y)=0$.

A vector field $W$ on a almost Hermitian manifold $M$ with almost complex structure $J$, is called contravariant almost analytic vector field, if the Lie derivative of $J$ with respect to $W$ vanishes identically for all vector field $X$ on $M$, i.e. $\left(L_{W} J\right)(X)=0$, this means that $[W, J X]=J[W, X]$. Therefore on a Kähler manifold, a vector field $W$ is contravariant almost analytic if and only if $\left(\bar{\nabla}_{J X} W\right)=J\left(\bar{\nabla}_{X} W\right)$ that is equivalent to $J\left(\bar{\nabla}_{J X} W\right)=-\bar{\nabla}_{X} W$. Now we have the following theorem as a direct result.

Theorem 3.6. Let $\widetilde{M}$ be a Kähler manifold with respect to both Levi-Civita connection $\bar{\nabla}$ and S-generalized connection $\breve{\nabla}$. If a vector field $W$ is contravariant almost analytic on $\widetilde{M}$ with respect to Levi-Civita connection $\bar{\nabla}$, then $W$ is also a contravariant almost analytic on $\widetilde{M}$ with respect to $\breve{\nabla}$.

Proof. By using (1.1) and linearity of $J$ we have,

$$
\breve{\nabla}_{J X} W-J\left(\breve{\nabla}_{X} W\right)=\bar{\nabla}_{J X} W-J\left(\bar{\nabla}_{X} W\right)+\varrho(W, X)
$$

Since $\widetilde{M}$ is a Kähler manifold with respect to connections $\bar{\nabla}$ and $\breve{\nabla}$, definition of contravariant almost analytic vector field implies that

$$
\bar{\nabla}_{J X} W-J\left(\bar{\nabla}_{X} W\right)=0 .
$$

So, the Corollary 3.5 gives us the result.

\section{References}

[1] N. S. Agashe and M. R. Chafle, A semi-symmetric non-metric connection in a Riemannian manifold, indian J. Pur Appl. Math., 23(1992), 399-409.

[2] E. Calabi, Construction and properties of some 6-dimensional almost complex manifolds, Trans. Amer. Math. Sot., 87(1958), 407-438.

[3] E. Calabi and B. Eckmann, A class of compact, complex manifolds which are not algebraic, Ann. of Math., 58(2)(1953), 494-500.

[4] S. K. Chauby and R. H. Ojha, On semi-symmetric non-metric and quarter-symmetric metric connection, Pur and Applied Mathematics Tensor N.S, 70(2)(2008), 202-213.

[5] Y. Doğru, On some properties of submanifolds of a Riemannian manifold endowed with a semi-symmetric non-metric connection, An. St. Univ. Ovidius Constanta, 19(3)(2011), 85-100.

[6] A. Friedmann, J. A. Schouten, Uber die geometrie der halbsymmetrischen ubertragungn, Math. Zeitschr, 21(1924), 211-233. 
[7] S. Golab, On semi-symmetric and quarter-symetric linear connections, Tensor N.S., 29(1975), 249-254.

[8] H. A. Hayden, Subspaces of space with torsion, Proc. London Math. Soc., 55(1994), $107-112$.

[9] Y. Liang, On semi-symmetric recurrent-metric connection, Tensor, 34(1932), 27-50.

[10] N. Pandey and B. B. Chaturvedi, semi-symmetric non metrics connections on a Kähler manifold, DGDS., 10(2008), 86-90.

[11] M. M. Tripathi, A new connection in a Riemannian manifold, Int. Electron. J. Geom., 1(1)(2008), 15-24. 